\title{
Insights into crystal growth pathways from isotopic tracers, molecular simulations, and theory
}

\author{
LAURA N. LAMMERS ${ }^{1,2^{*}}$, JENNIFER V. MILLS ${ }^{1}$, DONALD \\ J. DEPAOLO ${ }^{3}$ \\ ${ }^{1}$ Department of Environmental Science, Policy, and \\ Management, University of California, Berkeley \\ (*correspondence: Inlammers@berkeley.edu) \\ ${ }^{2}$ Energy Geoscience Division, Lawrence Berkeley National \\ Laboratory \\ ${ }^{3}$ Department of Earth and Planetary Science, University of \\ California, Berkeley
}

Controlling crystal growth from aqueous solution is of widespread interest for the selective extraction or immobilization of specific elements, for controlling scale formation during water treatment, and for advanced and biomimetic materials synthesis. However crystal growth reactions are multi-step processes that cannot be effectively modeled using simple kinetic theories.

In this talk I provide an overview of our recent progress using isotopic tracers combined with molecular simulations and kinetic theory to reconstruct the phenomenology of calcium carbonate growth over a wide range of supersaturations. The variation of stable calcium isotope fractionation factors as a function of aqueous supersaturation and solution stoichiometry (e.g. $\mathrm{Ca}^{2+}: \mathrm{CO}_{3}{ }^{2-}$ ) shed light on the dominant growth unit uptake pathways during calcite growth. Molecular dyanmics simulations results indicate that calcium ion desolvation can explain observed kinetic isotope fractionation, and these findings are integrated into an ion-byion kinetic model for calcite growth. When surface complexation is accounted for, the model captures the observed variability in calcium isotope fractionation with solution stoichiometry, suggesting that over the range of supersaturations studied (SI 0.5-1.2), calcite grows by a dominantly classical mechanism. Stable calcium isotope fractionation during classical calcite growth records the degree of kink exhange with the dissolved calcium pool, which is maximized at low growth rates and high $\mathrm{Ca}^{2+}: \mathrm{CO}_{3}{ }^{2-}$. At higher aqueous supersaturations, kinetic stable calcium isotope fractionation approaches a constant fractionation factor that is smaller in magnitude than for fast classical crystal growth, clearly signalling a transition to non-classical growth. Kinetic theories for growth by non-classical pathways and their impact on the isotope and trace element composition of the final crystal lag far behind classical theories and represent significant opportunities for future research. 